\title{
Association Between Work-Relate Musculoskeletal Disorder and Ergonomic Risk Factors Among Nursing Professionals in Ranya and Qaladiza Districts
}

\author{
Karwan Mahmood Khudhir \\ Department of Preventive Health, \\ Koya Technical Institute, Erbil, Iraq \\ karwan.khudhir@gmail.com
}

\author{
Kochar Khasraw Saleh \\ Department of Preventive Health, \\ Koya Technical Institute Erbil, Iraq \\ kocharkhasraw@gmail.com
}

\author{
Muhammed Saeed Qadir \\ Community Health Department, Sulaimani \\ Polytechnic University, Sulaimani, Iraq \\ bahast15@gmail.com
}

\author{
Kochr Ali Mahmood \\ Department of Preventive Health, \\ Koya Technical Institute, Erbil, Iraq \\ Kochrmahmood@yahoo.co.uk
}

\author{
Ahmad Azuhairi Ariffin \\ Department of Community Health, \\ Faculty of Medicine and Health Sciences \\ University Putra Malaysia, kuala lumpur, Malaysia \\ zuhairifin@upm.edu.my
}

\begin{abstract}
Work related musculoskeletal disorders (WRMSDs) represent one of the leading causes of occupational injury and disability among the professional nursing, due to the number and variety of risk factors associated with the work environment. This study was carried out to determine the prevalence of work related musculoskeletal disorders (WRMSDs) and its association with ergonomic risk factors. A selfadministered questionnaire and observational method was used to collect information from 420 individuals and then the data were computerized and analyzed by using SPSS version 21. The overall prevalence of WRMSDs among Ranya and Qaladiza districts nurses was $74 \%$. The neck pain was the most prevalent site of WRMSDs (48.4\%) compared to other body parts. Logistic regression analysis indicated that significant risk factors for WRMSDs symptoms were older nurses aged >39.5 years old (OR=3.076, 95\% CI: 1.200, 7.884), medium RULA risk level (OR= 255.096, 95\% CI: 24.078, 2702.681), very high RULA risk level (OR=151.675, 95\% CI: 17.536, 1311.891), low RULA risk level (OR=9.277, 95\% CI: 1.064, 80.893). Prevention strategies and health education which emphasizes on psychosocial risk factors and how to improve working conditions should be introduced.
\end{abstract}

Keywords: work-related musculoskeletal disorders, ergonomic risk factors, nurses, Rania

\section{INTRODUCTION}

Ergonomics is a science that seeks to improve employee performance and wellbeing in relation to job task, equipment and environment. Ergonomic application in the working environment will provide influence to the productivity of work, decrease health related problems, risk of injuries also increase job satisfaction and provide comfortable working condition while working. Nowadays, work related musculoskeletal disorders are common amongst the working population and one of the main problems encountered by ergonomists in diverse workplaces around the world. This type of work-related health problem can result in serious social impacts on both individuals and communities and may lead to work restriction, work-time loss, or consequently cause work leave [1]. WRMSDs is defined as an injury or disorder of the muscles, nerves, tendons, ligaments, joints, cartilage, or spinal discs, it is one of the most important occupational problems presently reported (Bureau, 2006) [2]. In the literature, various terms are used to describe musculoskeletal pain, disorders, symptoms, injury, trouble, discomfort and diseases [3]. The risk factors related to WRMSDs are recognized to comprise workplace activities such as manual handling, heavy lifting, strenuous task and work environment [4]. Health care work has been identified as high risk for developing WRMSDs [5]. According to the Australian data on work-related injuries (which include WRMSDs) indicate that the health and public facilities sector has one of the highest statements rates [6]. Most attention has been directed towards nursing as the major occupational group in the health care sector.

The profession of nursing is one of the most stressful and demanding careers in the present day, due to the number and variety of risk factors associated with the work environment. During a typical work day, nurses have to achieve numerous physically demanding occupations as well, such as transferring and repositioning patients between beds and chairs, lifting patients onto a bed, and frequently keeping bent forward or twisted postures. These type of work increases the activity of muscles and put greater stress on the ligament and joints, which can lead to fatigue and discomfort that put nurses at high risk for acute and cumulative WRMSDs [7]; the most frequently reported WRMSDs among nurses are low back pain, followed by neck and shoulder problems, and knee pain [8]. WRMSDs are reported to considerably impact quality of life, cause loss of work time or absenteeism, increase work restriction, transfer to another job, or disability than any other group of diseases with a significant financial toll on the individual, the organization and the society as a whole [9]. According to the United States Bureau of Labour Statistics (BLS, 2013) placed nursing among the professions with the highest rate of suffering from WRMSDs [10], studies in professional nursing on WRMSDs reported a prevalence rate of 70- 90\% [11, 8]. Among the listed disorders, low back pain was the highest prevalence, followed by neck, shoulder and extremity pain. 
However, many prevalence studies have reported MSDs among nurses; most research has been undertaken in the developed populations [11]. Iraq, in which this study was conducted, is a developing country to the best of our knowledge, no studies examining work related musculoskeletal disorders among nursing professionals, especially association between ergonomic risk factors and work related musculoskeletal disorders. Therefore, acknowledge gap exists in scientific literature regarding the prevalence of WRMSDs among professional nurses in Kurdistan Region-Iraq. The current study sought to fill this gap by investigating WRMSDs among nurses; the associated job risk factors and the coping strategies towards reducing the risk of development of WRMSDs among nurses from selected hospitals and health care centers.

\section{METHODS}

\subsection{Study design and sampling technique}

A cross sectional study administrated to nurses working in two general hospitals (Ranya and Qaladiza) and seven health care centers (Raparin, Kewarash, Chwarqurna, Zharawa, Sangasar, Shkarta and Sarwchawa) located in Raparin area which is consist of (Ranya and Pishdar district) of Sulaymaniyah city, Kurdistan region-Iraq. The required sample size was estimated using a formula for hypothesis testing of two group comparison [12] minimum calculated sample size was estimated at 92 . After adjusting 20\% for non-response and gender (multiplied by 2), the total sample size was 220 workers. Simple random sampling was applied to select 220 nurses based on the inclusive criteria. Nurses were invited to participate in the study with minimum one year experience working in the clinical area with no history of musculoskeletal disorders. Out of 220 selfadministered questionnaires, which were distributed among Nurses, 210 of them agreed to participate giving $95 \%$ respondent rate. At the end of the survey, only 192 questionnaires were analysed because 18 participants were excluded from the analysis (nurses who were pregnant at the time of study period and nurses with history of musculoskeletal disorders due to injuries). Data were collected between $10^{\text {th }}$ September to $30^{\text {th }}$ December, 2015.

The formula for the hypothesis testing of two groups that were compared was used (Lemeshow et al., 1990) [12].

$$
\begin{aligned}
& \mathrm{n}= \\
& \frac{\{Z 1-\alpha / 2 \sqrt{2 \tilde{P}(1-\tilde{P})}+Z 1-\beta \sqrt{P 1(1-P 1)+P 2(1-P 2)}\}^{2}}{(P 1-P 2)^{2}}
\end{aligned}
$$

The prevalence of MSDS among office workers in male was $54.4 \%$ and $73.9 \%$ in female in Tunisian hospital staff [13]

$$
\begin{aligned}
& \text { (). Where: } \\
& \mathrm{n} \text { = sample size }
\end{aligned}
$$

$$
\begin{aligned}
& \mathrm{P}=\text { prevalence } \\
& \mathrm{Z} 1-\alpha / 2=\mathrm{Z} \text { statistic for level of confidence of } \\
& 95 \%=1.96 \\
& \mathrm{Z} 1-\beta=\mathrm{Z} \text { statistic for } 80 \% \text { power }=0.842 \\
& \mathrm{P} 1=\text { prevalence of musculoskeletal disorders } \\
& \text { among males was } 54.4 \%=0.544
\end{aligned}
$$

$\& \mathrm{P} 2=$ prevalence of musculoskeletal disorders among females was $73.9 \%=0.739$$$
\tilde{\mathrm{P}}=\mathrm{P} 1+\mathrm{P} 2 / 2
$$$$
\mathrm{P}=\mathrm{P} 1+\mathrm{P} 2 / 2=0.641
$$

Minimum sample size: $\mathrm{n}=92$

After adjusting 20\% for non-response and gender (multiplied by 2), the total sample size was 220 workers.

\subsection{Data collection}

Data were collected from a questionnaire and direct observation. To glean subjective data a general questionnaire that covered the socio-demographic factors (age, gender) and occupational factors includes (job tenure, static work posture). Also, Standardized Nordic Questionnaire (SNQ) [12] was used to examine the musculoskeletal symptoms in any of nine anatomical body parts (neck and shoulder pain, upper back pain, low back pain, arm pain, knee and leg pain, ankle and foot pain) in the previous 12-months period as reported by the respondents.

The body movements of the participants during work tasks were observed by the first author in accordance with the Rapid Upper Limb Assessment (RULA) technique, which is known as a pen-paper observational method, is used to quantify and evaluate employee's potential risk exposure by assessing the posture, force and muscle activities and the scores were recorded. A separate RULA form was used for each employee and observation. In order to not interrupt the workers' concentration, the observations were conducted without allowing workers know about. However, the workers were told that they would be observed before the study was initiated. The development of RULA was done in three phases. The first was the development of the method for recording the working posture, the second phase involved the development of the scoring system, and the third was the development of the scale of action levels which provides a guide to the level of risk and need for action to conduct more detailed assessments. The scores were calculated for the posture of each body part. Score 1 shows the most neutral posture, score 7 indicates the worst posture. The scores of this tool consist of two groups: Score A includes upper arms, lower arms with wrists, Score B includes the trunk, neck with legs and Score C, which is called the grand Score (ranging from 1-7), are obtained by adding posture scores to the muscle use and force scores. A Grand Score 1-2 is an acceptable level score and negligible risk (action level 1); a Grand Score of 3-4 is low risk and change may be required (action level 2); a Grand Score 
of 5-6 is moderate risk, which requires more observation and change as soon as possible (action level 3 ) and if the score is greater than 6 it means that the risk is very high and there is need for immediate change (action level 4).

\subsection{Data analysis}

Statistical package for the social sciences (SPSS. 21) was used for statistical analysis. All variables were treated as categorical and nonnormally distributed. Descriptive characteristics of the respondents were calculated as (frequency, percentage, median, and interquartile range). Chi square test was used to determine the association between categorical variables and musculoskeletal disorders. Totally significant variables (at $P<0.05$ ) were included in subsequent multivariable logistic regression to estimate the adjusted odds ratios of factors associated with musculoskeletal disorders.

\subsection{Ethical consideration}

Ethics approval was obtained from the Ethics Committee of Koya Technical Institutes in August 2015 (ref: KTI 12512). Before collecting data, approval was also obtained from respective directorate of public hospitals plus health care centers and a written letter of consent was obtained from each participant. The respondents were informed about the purpose of the study and that participating in this study was voluntary. Furthermore, the participants were told that their answers would be kept confidential and would only be used for research purposes.

\section{RESULTS}

A total 210 (response rate $=95 \%$ ) subjects participated in the study. Details of the socio-demographic, occupational factors are presented in Table 1, which, shows that the age distribution of the nurses is between 21 and 62 years and they are distributed into two groups, with $50.5 \%$ in the older group (>39.5 years) and $49.5 \%$ in the younger group $(\leq 39.5)$, with median of age at 39.5 years (IQR=9.75). Also, majority of Kurdistan nurses were female $(57.3 \%)$. Based on the occupational factors, a majority of the nurses $(61.5 \%)$, had history of wok $\leq$ 10 years; standing equal or less than two hours during working days $(52.6 \%)$ and $(79.7 \%)$ sitting equal or more than three hours. The median of job tenure was ten years ( $\mathrm{IQR}=9.0$ ), as for standing median was 2 hours/working days $(\mathrm{IQR}=2.0)$ and median of sitting was 2 hours/working days $(\mathrm{IQR}=3.0)$. As for the RULA risk assessment for awkward posture majority of the Kurdistan nurses were in very high risk level of RULA, where change needs to be implemented now. Median RULA risk level score was 3 (IQR=2).

Table 1. Demographic and occupational factors nurses who participated in the study

\begin{tabular}{|c|c|c|c|c|c|}
\hline Factors & \multirow[t]{2}{*}{ Frequency $(\%)$} & \multirow[t]{2}{*}{ Median (IQR) } & \multicolumn{2}{|c|}{ MSDs } & \multirow[t]{2}{*}{$P$ value } \\
\hline & & & Yes & No & \\
\hline Age groups (years) & & $39.5(9.75)$ & & & 0.007 \\
\hline \begin{tabular}{l|l} 
& $\leq 39.5$ \\
\end{tabular} & $95(49.5)$ & & 62 & 33 & \\
\hline$>39.5$ & $97(50.5)$ & & 80 & 17 & \\
\hline Gender & & & & & 0.77 \\
\hline \begin{tabular}{l|l} 
& Male \\
\end{tabular} & $85(44.3)$ & & 62 & 23 & \\
\hline Female & $107(57.3)$ & & 80 & 27 & \\
\hline Job tenure & & $10(9.0)$ & & & 0.14 \\
\hline $\mid$\begin{tabular}{l|l} 
& $\leq 10$ years
\end{tabular} & $118(61.5)$ & & 83 & 35 & \\
\hline \begin{tabular}{l|l} 
& $\geq 11$ years \\
\end{tabular} & $74(38.5)$ & & 59 & 15 & \\
\hline Static posture (standing) & & $27(6.9)$ & & & 0.12 \\
\hline \begin{tabular}{l|l} 
& Standing $(\leq 2)$ \\
\end{tabular} & $101(52.6)$ & & 70 & 31 & \\
\hline Standing $(\geq 3)$ & $91(47.4)$ & & 72 & 19 & \\
\hline Static posture (sitting) & & & & & 0.34 \\
\hline \begin{tabular}{l|l} 
& Sitting $(\leq 2)$ \\
\end{tabular} & $55(20.3)$ & & 82 & 25 & \\
\hline Sitting $(\geq 3)$ & $216(79.7)$ & & 60 & 25 & \\
\hline Risk Level & & $3(2)$ & & & $<0.001$ \\
\hline \begin{tabular}{l|l} 
& Negligible risk \\
\end{tabular} & $18(9.4)$ & & 1 & 17 & \\
\hline Low risk & $32(16.7)$ & & 12 & 20 & \\
\hline Medium risk & $49(25.5)$ & & 46 & 3 & \\
\hline Very high risk & $93(48.4)$ & & 83 & 10 & \\
\hline
\end{tabular}


Table 2 presents the overall prevalence of WRMSDs among Kurdistan nurses in the past 12 months was $74 \%$. In descending order, the body parts reported to be subjected to musculoskeletal discomfort were the neck pain $94(48.5 \%)$, followed by shoulder, 74 (38.5\%), upper back $72(37.5 \%)$, feet/ankle 57 (29.7\%), lower back 51(26.6\%), thigh/ hip 50 (26\%), Wrists/ Hands 47 (24.5), while the lowest rate were found to be in knee 43 $(22.4 \%)$ and elbows $32(16.7 \%)$.

Table 2. Twelve-month prevalence of WRMSDs according to body region

\begin{tabular}{|l|l|}
\hline Body region & No. $(\%)$ \\
\hline Neck & $93(48.4)$ \\
\hline Shoulders & $74(38.5)$ \\
\hline Elbows & $32(16.7)$ \\
\hline Wrists/ Hands & $47(24.5)$ \\
\hline Upper back & $72(37.5)$ \\
\hline Lower back & $51(26.6)$ \\
\hline Thighs/ Hips & $50(26)$ \\
\hline Knee & $43(22.4)$ \\
\hline Feet /Ankle & $57(29.7)$ \\
\hline
\end{tabular}

The inferential analysis in Table 1 showed that there were significant associations between age, RULA risk level and MSDs $(P<0.05)$ among Kurdistan nurses, but there are no significant associations shown by chi square ( $P$ value) between WRMSDs with gender, Job tenure, standing and sitting work posture $(P>0.05)$.

In addition, independent variables with $\mathrm{P}<0.05$ in the inferential analysis were simultaneously analyzed by multiple logistic regressions (Table 2). The results showed that nursing professionals with older age group and RULA risk level (low, medium and high) were found to be significantly associated with WRMSDs. Nurses who were in older age group ( $>39.5$ years old) were found to have 3 times (95\% CI: 1.200 to 7.884) higher risk of WRMSDs as compared to younger group nurses. In the same way, nurses in the medium RULA risk level were found to have 255 times (95\% CI: 24.078 to 2702.681) higher risk of WRMSDs as compared to the nurses who were in negligible risk. Nurses in the very high RULA risk level were found to have 151 times (95\% CI: 17.536 to 1311.891) higher risk of WRMSDs as compared to those who were in negligible risk. In addition, nurses in the low RULA risk level were found to have 9 times (95\% CI: 1.064 to 80.893 ) higher risk of WRMSDs as compared to those who were in negligible risk. In this modeling, the Negelkerke $\mathrm{R}^{2}$ showed that about $54.8 \%$ of the variation of WRMSDs was explained by independent variables

Table 3. Multiple logistic regressions of predictors of musculoskeletal disorders

\begin{tabular}{|c|c|c|c|c|c|}
\hline Variables & $\mathbf{B}$ & S.E & Sig. & Adjusted OR & $95 \% \mathrm{CI}$ \\
\hline \multicolumn{6}{|l|}{ Age } \\
\hline$\leq 39.5$ & - & - & - & 1 & - \\
\hline$>39.5$ & 1.124 & 0.480 & 0.019 & 3.076 & $1.200,7.884$ \\
\hline \multicolumn{6}{|l|}{ Risk Level } \\
\hline \begin{tabular}{l|l} 
& Negligible risk
\end{tabular} & - & - & - & 1 & - \\
\hline Low risk & 2.228 & 1.105 & .044 & 9.277 & $1.064,80.893$ \\
\hline Medium risk & 5.542 & 1.204 & $<0.001$ & 255.096 & $\begin{array}{c}24.078, \\
2702.681\end{array}$ \\
\hline Very high risk & 5.022 & 1.101 & $<0.001$ & 151.675 & $\begin{array}{c}17.536, \\
1311.891\end{array}$ \\
\hline
\end{tabular}

\section{DISCUSION}

A high prevalence of WRMSDs is (74\%) was found among Kurdistan nurses. Our results is close with previous study among Malaysian public hospital nurses, who reported prevalence of WRMSDs (73.24\%) [14], but also higher compared to other study findings, which reported the prevalence of WRMSDs (57.2\%) among nurses working in governmental health institutions of Gondar town, Ethiopia. [15] Nevertheless, as compared to nursing employee in other Asia countries, the prevalence of WRMSDs was found to be much lower, (78.0\%-94.6\%). [16]

In this study the highest prevalence of WRMSDs were found in neck with prevalence of $(48.5 \%)$, followed by shoulder $(38.5 \%)$ and upper back 72 $(37.5 \%)$. These findings are consistent with the study conducted among Malaysian public hospital nurses in which neck pain, upper back were the most common and 
mainly occurring prevalence of MSDs. [12] Whereas in the earlier study conducted in Japan among nurses most prevalent of WRMSDs reported was that of shoulder (71.9\%), followed by low back (71.3\%), neck (54.7\%), and upper back (33.9\%). [17] In the current study age was seen to be important factor in WRMSDs. The results of this study are in accordance with the most of the previous researches done. [8] Besides, this study shows that the prevalence of WRMSDs is $(82 \%)$ and much higher among the older group nurses when compared to younger age group nurses (65\%). Possible explanation for the increase in WRMSDs prevalence among older group workers compared to the younger group could be ageing, biological changes; biological in nature due to the ageing process such as, degenerative changes that occur in muscles, tendons, ligaments, nerves, and joints. Based on these two important findings among the nurses who were in older age, and the fact that the prevalence of WRMSDs was higher among them, there is an essential necessary for this problem to be tackled completely by organize programs that promote physical exercise and training in ergonomics.

However, the result of this study show that there is no statistically significant association was found between gender and WRMSDs, the prevalence of WRMSDs is slightly higher among female nurses than male nurses ( $75 \%$ vs $73 \%$ respectively). This finding is in line with the study conducted among Denmark nurses. [14] Intrinsic risk factors may be playing an important role in the onset and deterioration of MSDs among women. Several intrinsic factors can be altered whereas others, such as genetic predisposition, cannot. Also, a number of studies, for example, have noted that a higher prevalence of work related musculoskeletal disorder among working women may be related to the fact that women are still generally responsible for doing the majority of housework. [18] Similarly, there were not significant association found between WRMSDs and job tenure, standing and sitting work posture.

As indicate previously, RULA allows a rapid assessment of work-related loads of workers musculoskeletal systems to posture, muscle use and the force exerted in performing their tasks. From the observation, nurses were involved with awkward working posture; manual material handling, lower back bending forward as transferring and repositioning patients between beds and chairs, lifting patients onto a bed, and frequently keeping bent forward or twisted postures for long hours without any adequate rest were common possible ergonomic cause to WRMSDs. Based on the RULA risk assessment in this study majority of the Kurdistan nurses were in very high risk level (48.4\%) of exposure to musculoskeletal risk and besides shows statistically significant association between RULA risk level and WRMSDs obtained from SNQ ( $P$ $<0.001)$. This indicates that nurses are exposed to extreme bending and twisting in various parts of their bodies (awkward posture), also using force and requiring muscle strength while carrying out their work related tasks. When the nurses did the work, they repetitively moved their upper and lower limbs in manners that were inconsistent with natural anatomical postures. This led to excessive workloads for both their muscles and tendons as the nurses frequently required maintaining their body balance. Altogether these ergonomic factors are acknowledged causative factors for WRMSD. Also, Multiple logistic regression showed that the nurses in low, medium and higher RULA risk levels were found to have $9,255,151$ times respectively higher risk of WRMSDs as compared to those who were in negligible RULA risk level. This study finding is in agreement with the previous studies conducted by Munabi et al. [19], among nursing professionals in Uganda that had demonstrated high RULA risk level was related to WRMSDs. With regards to the study findings, it is important to note that the current research was of crosssectional design, which may not represent a causal association between risk factors studied with WRMSDs. Also, the twelve month prevalence of each body part and working conditions were based on self-reporting questionnaire to collect data, and, as a result, recall bias cannot be ruled out. The outcomes should therefore be seen as provisional ones that offer a starting point for further research, including cohort studies that are better able to recognise contributing factors that are related with WRMSDs.

Future work related musculoskeletal disorders prevention efforts should emphasize the need to reduce the RULA Grand Score via limit repetitive motions, awkward postures, and safe resident lifting program that incorporate mechanical lifting equipment can be highly effective in reducing nurse's exposure to heavy loads and awkward working postures that contribute to back and other musculoskeletal disorders.

\section{ACKNOWLEDGEMENTS}

The authors gratefully acknowledge Riparian Director of Preventive Health, Raparin Director of General Health, Kurdistan Ministry of Health, Directors the Hospital and Health care Centers and their staff who contributing in this study.

\section{DECLARATION OF CONFLICTING INTERESTS}

The author declared no potential conflicts of interest with respect to the research, authorship, and/or publication of this article.

\section{FUNDING}

The author received no financial support for the research, authorship, and/or publication of this article.

\section{REFERENCES}

[1] MS. Forde, L. Punnett, DH. Wegman, "Pathomechanisms of work-related musculoskeletal disorders:conceptual issues," Ergonomics, vol.45, pp. 619-630, 2002. 
[2] Bureau of Labor Statistics, "Non-fatal occupational injuries and illness requiring days away from work," 8 September 2016.[Online]. Available: http://www.bls.gov/iif/oshwc/osh/case/osnr0015.pd f. [Accessed: 2013].

[3] AM. Scuffham, SJ. Legg, EC. Firth, MA. Stevenson, "Prevalence and risk factors associated with musculoskeletal discomfort in New Zealand veterinarians," Applied ergonomics, vol. 41, pp. 444-453, 2010.

[4] J. Smedley, P. Egger, C. Cooper, D. Coggon, "Prospective cohort study of predictors of incident low back pain in nurses," Bmj, vol. 314 , pp.1225, 1997.

[5] JM. Tullar, S. Brewer, BC. Amick, E. Irvin, Q. Mahood, LA. Pompeii, et al., "Occupational safety and health interventions to reduce musculoskeletal symptoms in the health care sector," Journal of occupational rehabilitation, vol. 20, pp.199-219, 2010 .

[6] Safe Work Australia, "Compendium of Workers' Compensation Statistics Australia 2010e11," Canberra, Australia, 10. March 2016. [Online]. Available:

http://www.safeworkaustralia.gov.au/sites/swa/abo ut/publication/pages /compendium-2010-11. [Accessed: 2012].

[7] M. Jaworek, T. Marek, W. Karwowski, C. Andrzejczak, AM. Genaidy, "Burnout syndrome as a mediator for the effect of work-related factors on musculoskeletal complaints among hospital nurses," International Journal of Industrial Ergonomics, vol. 40, pp. 368-75, 2010

[8] AM. Trinkoff, JA. Lipscomb, J. Geiger- Brown, B. Brady, "Musculoskeletal problems of the neck, shoulder, and back and functional consequences in nurses," American journal industrial medicine, vol. 41, pp. 170-8, 2002.

[9] DB. Anap, C. Iyer, K. Rao, "Work related musculoskeletal disorders among hospital nurses in rural Maharashtra, India: a multi centre survey," International Journal of Research in Medical Sciences, vol. 1, pp. 101-107, 2017.

[10] Bureau of Labor Statistics, "Non-fatal occupational injuries and illness requiring daysaway from work," 8. Sep, 2015. [Online]. Available: http://www.bls.gov/iif/oshwc/osh/case/osnr0015.pd f. [Accessed: 2013].
[11] DR. Smith, N. Wei, L. Kang, RS. Wang, "Musculoskeletal disorders among professional nurses in mainland China," Journal of Professional Nursing, vol. 20, pp. 390-395, 2004.

[12] S. Lemeshow, D. Hosmer, J. Klar, S. Lwanga, \& World Health Organization, "Adequacy of sample size health studies, 1990.

[13] A. Jella, H. Lajili, S. Boudokhane, H. Migaou, S. Maatallah, \& Z. Frih, "Musculoskeletal disorders among Tunisian hospital staff: Prevalence and risk factors," The Egyptian Rheumatologist, vol. 35, pp. 59-63, 2013.

[14] NA. Amin, R. Nordin, QK. Fatt, RM. Noah, J. Oxley, "Relationship between Psychosocial Risk Factors and Work-Related Musculoskeletal Disorders among Public Hospital Nurses in Malaysia," Annals of occupational and environmental medicine, vol. 26, pp. 23, 2014.

[15] A. Yitayeh, S. Fasika, S. Mekonnen, M. Gizachew, "Work related musculoskeletal disorders and associated factors among nurses working in governmental health institutions of Gondar town, Ethiopia," Physiotherapy, vol. 101, pp. e1694, 2015.

[16] YC. Chung, CT. Hung, SF. Li, HM. Lee, SG. Wang, SC. Chang, et al., "Risk of musculoskeletal disorder among Taiwanese nurses cohort: a nationwide population-based study," BMC musculoskeletal disorders IV, vol. 14, pp. 144, 2013.

[17] CD. Rasmussen, AK. Larsen, A. Holtermann, K. Søgaard, MB. Jørgensen, "Adoption of workplaces and reach of employees for a multifaceted intervention targeting low back pain among nurses' aides," BMC medical research methodology, vol. 14, pp. 60, 2014.

[18] L. Punnett, DH. Wegman, "Work-related musculoskeletal disorders: the epidemiologic evidence and the debate," Journal of electromyography and kinesiology, vol. 14, pp. 13-23, 2004.

[19] IG. Munabi, W. Buwembo, DL. Kitara, J. Ochieng, ES. Mwaka, "Musculoskeletal disorder risk factors among nursing professionals in low resource settings: a cross-sectional study in Uganda,” BMC nursing, vol. 13, pp. 7, 2014. 